\title{
Reconfigurable Manufacturing Implementation
}

\author{
Basel Alsayyed*, Khalifa Harib \\ Department of Mechanical Engineering, United Arab Emirates University Al Ain P.O. Box 15551, UAE \\ *Corresponding author: balsayyed@uaeu.ac.ae
}

Received October 24, 2014; Revised November 01, 2014; Accepted November 04, 2014

\begin{abstract}
As trade competition is getting fiercer, innovation in all areas of product realization is becoming more of a need than a want. In this paper, the authors present an innovative idea for an actual implementation of reconfigurable manufacturing, where a FlexDie is designed to manufacture sheet metal parts. The idea is to mimic the representation of graphics and text on a screen using pixels. The developed FlexDie in this paper consists of the two halves that are similar in design. It comprises pixel like pins assembled next to each other in the XY plane. The pins' heights in the Z-direction are designed to be adjustable to yield a desired topology. Each pixel can be altered and locked at a certain Z- coordinate. A prototype is developed to test the concept and produce preliminary results. The developed prototype consists of two-half dies, each having 8x8 square pixels, each of about 10x10 square mm. In the implemented design the Z-coordinates of the pixels are controlled and locked at desired heights via an array of screws. Sample parts consisting of three-dimensional metal sheets were manufactured using the developed FlexDie. This implementation opens the door for a new way of reconfigurable manufacturing, where the hardware is designed to be programmable.
\end{abstract}

Keywords: reconfigurable manufacturing, CAD/CAM integration, FMS, flexible die, group technology, sustainable manufacturing

Cite This Article: Basel Alsayyed, and Khalifa Harib, "Reconfigurable Manufacturing Implementation." American Journal of Mechanical Engineering, vol. 2, no. 5 (2014): 147-150. doi: 10.12691/ajme-2-5-4.

\section{Introduction}

As market is pushing for more variety and less quantity, innovative solutions are needed to keep cost and quality in check. Reconfigurable manufacturing is the answer. A real life implementation of reconfigurable manufacturing is included in this paper for sheet metals manufacturing. Flexible die (FlexDie) is an innovative idea for sheet metal and plastic forming processes that is becoming inline with the current interest in developing sustainable manufacturing processes. To the contrary of a fixed die that is used to produce parts of the same design, with FlexDie various parts with different designs can be produced using the same die. The process can be automated and programmable to form a variety of similar products using the same die. The topology of the die surface could be programmed and constructed via discrete surfaces formed from pixels instead of a fixed topology in traditional sheet metal forming. The benefits of such approach are multi fold. It considerably reduces the cost of the die making as well as all the associated cost of maintaining and storing unused dies. Reducing die development time for newly designed products is also highly attractive. Currently the general trend in product development is to go from mass production to batch and craft shop quantities with large variety of products [1]. With this trend the die making and maintaining problem is becoming more challenging. Flexible die with adjustable topology is explored by many researchers recent years.
Peng used NURBSS surface extension to design the blend of the surface between the blank holder and the workpiece. Introducing Multi point forming [2,3] is a promising advanced flexible manufacturing approach to handle the variety of products by dividing the surface of the die into pins [4]. Sun studied the principals of flexible and rigid blank holders forming, a blank holder was placed above the workpiece and another under the workpiece, different types of blank holders resulted in sharply different results [5]. Wrinkles and dimples are the major forming defects in this process [6]. Pins used in previously related work are of circular sections; in this paper we are using the squared section pins, which will give a full cover of the effective die area. The behavior of the deformed material is observed and further work is undergoing to refine the design of the pixels and their orientation.

\section{Method Description}

In this paper the idea of multi-point forming is introduced using square section pixels. Each pixel is controlled independently from the other pixels. A prototype of a FlexDie was manufactured in our lab with an $8 \times 8$ array of pixels in both the upper and the lower halves of the die. In this arrangement, the pins, viewed as pixels, are setup next to each other in the XY- plane. Figure 1 below shows the pixels orientation, dimensions and notation. 


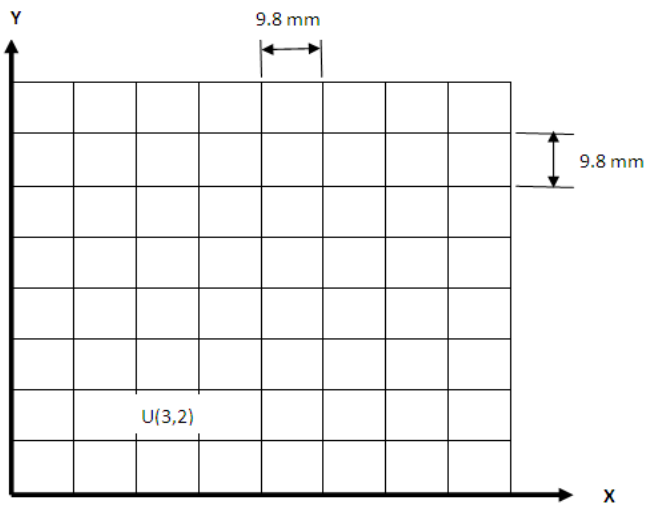

Figure 1. pixels dimensions, orientation and notation

Each pixel is adjustable in the Z-axis direction independently from the other pixels, and is referred to in terms of its location in the upper die with the notation $\mathrm{U}(\mathrm{x}, \mathrm{y})$ location, and $\mathrm{L}(\mathrm{x}, \mathrm{y})$ for the lower part. Example is shown in Figure 1 for the upper part. In the implemented design, each pixel represents a $9.8 \times 9.8 \mathrm{~mm}$ squared section steel bar with a $40 \mathrm{~mm}$ length. Each pixel is supported by an M-8 bolt that controls its Z-location.

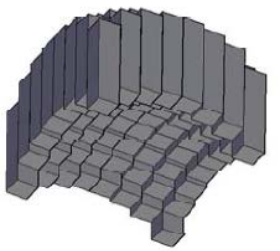

(a)

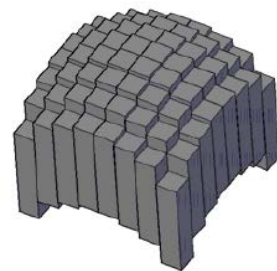

(b)
Figure 2. Example for pins' arrangements for a concaved part, (a) upper and (b) lower die halves

The pixels movement along the Z-axis is coordinated between the lower and upper set of pixels such that each pixel in each set corresponds to another pixel from the other set. The pixels are referred to in terms of their $\mathrm{x} \& \mathrm{y}$ locations. Each pixel in the upper and lower die part has the same magnitude of $\mathrm{Z}$ coordinate but in opposite directions. Hence, the upper and lower parts of the die will take the desired shape of the final part. An example of a concaved forming is used here and is shown in Figure 2.

The die was designed and manufactured in the manufacturing lab at the UAE University. A section drawing of the die is shown in Figure 3. It shows the upper and lower parts of the die as well as the pixels orientation in both sections. The pixels are lined up in a matrix where each pixel is adjusted by an individual bolt. This allows for independent adjustment of the pixels' heights. This independent adjustment can theoretically produce large number of shapes. The upper and lower pixel's heights will complement each other to the flat level of the pixels. The heights of all pixels in both the upper and lower die parts have to be calculated based on the part to be manufactured profile. The process of calculating the pixels' heights can be automated and integrated with standard Computer Aided Engineering tools.

\section{Example and Results}

An example of concaved part using a 8x8 FlexDie is employed to demonstrate the idea. The calculated heights using MS Excel are shown in Table 1 below for the upper and lower die halves. The surface of the part is an upper surface of a sphere with radius $\mathrm{R}$, and is defined by Equations (1), (2), and (3) as indicated below.

$$
\begin{aligned}
& x=R \cos \theta \cos \phi \\
& y=R \cos \theta \sin \phi \\
& z=R \sin \theta
\end{aligned}
$$

For a given radius $\mathrm{R}$ and a set of coordinates $(\mathrm{x}, \mathrm{y})$, the first two equations in (1) can be solved for $\theta$, which is then used in the third equation to calculate the pixel height $\mathrm{z}$.

Based on the calculated heights for all pixels, the pins' heights are adjusted individually using the corresponding screws and thus controlling pixels' desired heights. Figure 4 shows the back view of the die. The setup of the distances is done in reference to the zero level of all the pixels. The upper pixels and lower pixels are adjusted to equal distances in opposite directions as shown in Figure 5 (a) and (b). The pixels are allowed to slide freely as the bolts behind them are adjusted to raise or lower each individual pixel. The setup in Figure 5 (a) and (b) was done for a $0.5 \mathrm{~mm}$ thick aluminum sheet. The dimensions of the effective area is the summation of the individual pixels in both $\mathrm{x}$ and $\mathrm{y}$ direction. As the heights are adjusted to different levels, the resulting part will be different. The number of bolts for this setup is 64 for each part, which make it time consuming to do the setup manual. In the next version of this die, the setup will be automated to reduce the setup time and increase accuracy. After adjusting the heights of all pixels for the example part, the die upper and lower parts are shown in Figure 5.

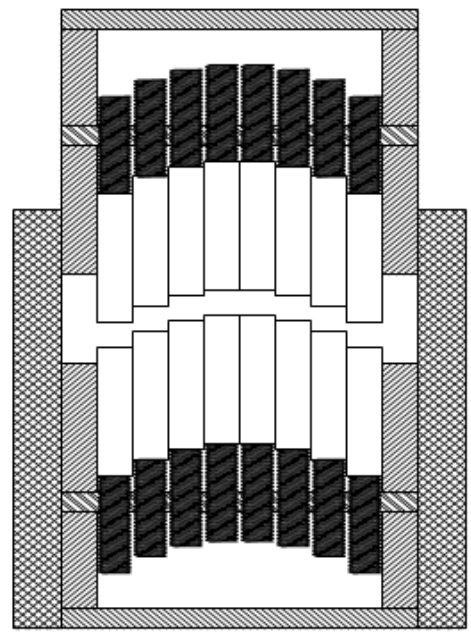

Figure 3. Schematic drawing for a section along the upper and lower die parts

Once the heights of the bolts of both parts of the die are setup and the workpiece is prepared and placed in the die; the die is pressed on the workpiece to form it. A manual press was used to form a $0.5 \mathrm{~mm}$ and a $0.8 \mathrm{~mm}$ sheet metal parts. Pictures of the resulting parts are shown in Figure 6. It was noticed that the $0.5 \mathrm{~mm}$ part showed some fractures especially near the side pixels, Figure 6 (a). Investigation of those fractures revealed that the height of the pixels near the edges needs to be less to reduce the individual pixel drawing distance. The 
heights were adjusted at one of the edges that showed the max fracture, and the fractures reduced substantially. More investigation is undergoing to optimize on an adjusting factor to the calculated height shown in Table 1 (a) and (b) above. For the $0.8 \mathrm{~mm}$ sheet, fractures were much less.

Table 1. (a) and (b) shows calculated heights of the pins in the two halves of the die for the considered example

(a)
\begin{tabular}{|c|c|c|c|c|c|c|c|}
\hline 0 & 7.9 & 12.4 & 14.5 & 14.5 & 12.4 & 7.9 & 0 \\
\hline 7.9 & 14.5 & 18.5 & 20.4 & 20.4 & 18.5 & 14.5 & 7.9 \\
\hline 12.4 & 18.5 & 22.2 & 23.9 & 23.9 & 22.2 & 18.5 & 12.4 \\
\hline 14.5 & 20.4 & 23.9 & 25.6 & 25.6 & 23.9 & 20.4 & 14.5 \\
\hline 14.5 & 20.4 & 23.9 & 25.6 & 25.6 & 23.9 & 20.4 & 14.5 \\
\hline 12.4 & 18.5 & 22.2 & 23.9 & 23.9 & 22.2 & 18.5 & 12.4 \\
\hline 7.9 & 14.5 & 18.5 & 20.4 & 20.4 & 18.5 & 14.5 & 7.9 \\
\hline 0 & 7.9 & 12.4 & 14.5 & 14.5 & 12.4 & 7.9 & 0 \\
\hline
\end{tabular}

(b)

\begin{tabular}{|c|c|c|c|c|c|c|c|}
\hline 26 & 18.1 & 13.6 & 11.5 & 11.5 & 13.6 & 18.1 & 26 \\
\hline 18.1 & 11.5 & 7.5 & 5.6 & 5.6 & 7.5 & 11.5 & 18.1 \\
\hline 13.6 & 7.5 & 3.8 & 2.1 & 2.1 & 3.8 & 7.5 & 13.6 \\
\hline 11.5 & 5.6 & 2.1 & 0.4 & 0.4 & 2.1 & 5.6 & 11.5 \\
\hline 11.5 & 5.6 & 2.1 & 0.4 & 0.4 & 2.1 & 5.6 & 11.5 \\
\hline 13.6 & 7.5 & 3.8 & 2.1 & 2.1 & 3.8 & 7.5 & 13.6 \\
\hline 18.1 & 11.5 & 7.5 & 5.6 & 5.6 & 7.5 & 11.5 & 18.1 \\
\hline 26 & 18.1 & 13.6 & 11.5 & 11.5 & 13.6 & 18.1 & 26 \\
\hline
\end{tabular}

The pictures in Figure 7 (a) and (b) show a reduction in part fracture when using a sheet blanket to compensate for the rough pixels' size and sharp edges. The blanket used is $0.5 \mathrm{~mm}$ thick of textile material. The results show smooth consistent curvature of the part.

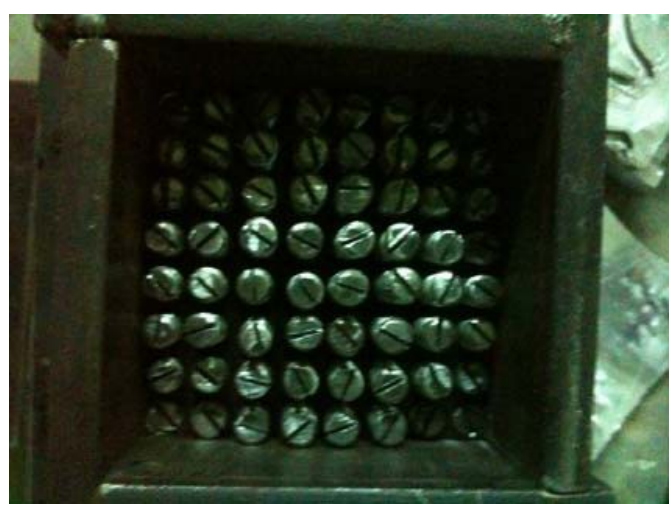

Figure 4. The screw-side of the die

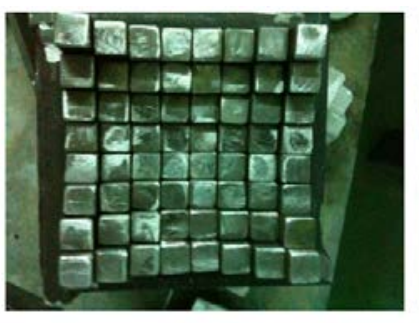

(a)

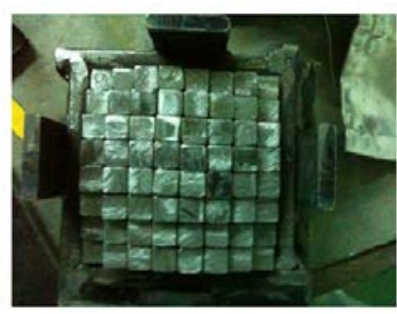

(b)
Figure 5. (a) and (b) upper and lower parts of the die for the example part

\section{Conclusion and Future Work}

In this paper a reconfigurable manufacturing concept has been demonstrated through the idea of using a flexible die to manufacture variety of curved metal sheet parts. In FlexDie the authors examined squared section pins controlled by a set of screws. Although in the presented design we limited ourselves to using pins with square cross section and flat square face, the used pins could be selected from assortment of square pins with inclined faces with standard angles (e.g. $15^{\circ}, 30^{\circ}$, $\left.45^{\circ}, 60^{\circ}, 75^{\circ}\right)$. This added flexibility will increase the smoothness of the generated curved die topology.

The process is done manually in the presented experiment where the deformation and shape of the workpiece is controlled manually. The preliminary results obtained are promising and work is continuing in our lab to improve the resolution and capability of the process. More applications are understudy where the control of the pixels is done via the CAD system. Work is undergoing for manufacturing different versions of the FlexDie for different applications. Also, in the next generation of this FlexDie, the CAD model will be post processed to send a signal to a PLC controlled mechanism or robot to typeset pins and to control their Zcoordinates and lock then in place.

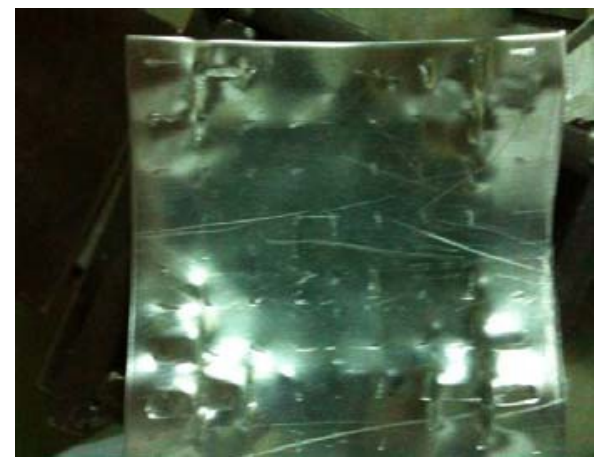

Figure 6. A part manufactured using the FlexDie

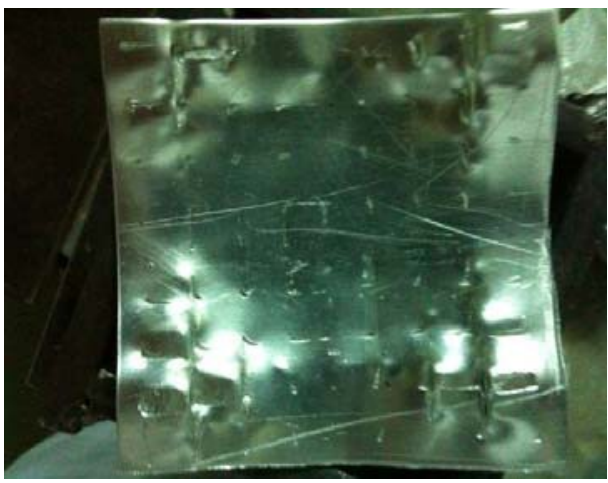

Figure 7. A part produced using a blanket to smooth the sharp edges

\section{References}

[1] L. Peng, X. Lai, M. Li, Transition surface design for blank holder in muti-point forming, et al. / International Journal of Machine Tools \& Manufacture 46 (2006) 1336-1342

[2] M. Li, Y. Liu, et al., Multi-point forming: a flexible manufacturing method for a 3-d surface sheet, J. Mater. Process. Technol. 87 (1999) 277-280. 
[3] M.Z. Li, Z.Y. Cai, Z. Sui, Q.G. Yan, Multi-point forming technology for sheet metal parts, J. Mater. Process. Technol. 129 (1-3) (2002) 333-338.

[4] Z.R. Qian et al. / The analyse on the process of multi-point forming for dish head Journal of Materials Processing Technology 187-188 (2007) 471-475.
[5] G. Sun et al. / Study of blank-holder technology on multipoint forming of thin sheet metal, Journal of Materials Processing Technology 187-188 (2007) 517-520.

[6] M.Z. Li, Z.Y. Cai, Z. Sui, et al., Multi-point forming technology for sheet metal [J], J. Mater. Process. Technol. 129 (2002) 333-338. 